\title{
Harmonic bases for generalized coinvariant algebras
}

\author{
Brendon Rhoades* Tianyi Yu Zehong Zhao \\ Department of Mathematics \\ University of California, San Diego \\ La Jolla, CA, 92093, U.S.A. \\ \{bprhoades, tiy059, zez045\}@ucsd.edu
}

Submitted: May 26, 2020; Accepted: Oct 8, 2020; Published: Oct 30, 2020

(C) The authors. Released under the CC BY-ND license (International 4.0).

\begin{abstract}
Let $k \leqslant n$ be nonnegative integers and let $\lambda$ be a partition of $k$. S. Griffin recently introduced a quotient $R_{n, \lambda}$ of the polynomial ring $\mathbb{Q}\left[x_{1}, \ldots, x_{n}\right]$ in $n$ variables which simultaneously generalizes the Delta Conjecture coinvariant rings of Haglund-Rhoades-Shimozono and the cohomology rings of Springer fibers studied by Tanisaki and Garsia-Procesi. We describe the space $V_{n, \lambda}$ of harmonics attached to $R_{n, \lambda}$ and produce a harmonic basis of $R_{n, \lambda}$ indexed by certain ordered set partitions $\mathcal{O} \mathcal{P}_{n, \lambda}$. The combinatorics of this basis is governed by a new extension of the Lehmer code of a permutation to $\mathcal{O P}_{n, \lambda}$.
\end{abstract}

Mathematics Subject Classifications: 05E05, 05E10, 05E40

\section{Introduction}

In his Ph.D. thesis [5], Sean Griffin introduced the following remarkable family of quotients of the polynomial ring $\mathbb{Q}\left[\mathbf{x}_{n}\right]:=\mathbb{Q}\left[x_{1}, \ldots, x_{n}\right]$ in $n$ variables. Given a subset $S \subseteq[n]:=$ $\{1,2, \ldots, n\}$ and $d \geqslant 0$, let $e_{d}(S)$ be the degree $d$ elementary symmetric polynomial in the variable set $\left\{x_{i}: i \in S\right\}$. For example, we have

$$
e_{2}(1457)=x_{1} x_{4}+x_{1} x_{5}+x_{1} x_{7}+x_{4} x_{5}+x_{4} x_{7}+x_{5} x_{7} .
$$

By convention, we set $e_{d}(S)=0$ whenever $|S|<d$.

For $k \geqslant 0$, in this paper we use the term partition of $k$ to mean a weakly decreasing sequence $\lambda=\left(\lambda_{1} \geqslant \cdots \geqslant \lambda_{s}\right)$ of nonnegative integers with $\lambda_{1}+\cdots+\lambda_{s}=k$. We write $|\lambda|=k$ or $\lambda \vdash k$ to mean that $\lambda$ is a partition of $k$ and call $s$ the number of parts of $\lambda=\left(\lambda_{1}, \ldots, \lambda_{s}\right)$. We also write $\ell(\lambda)$ for the number of nonzero parts of $\lambda$. For example, if $\lambda=(4,2,2,0,0)$ we have $\lambda \vdash 8$, the partition $\lambda$ has 5 parts, and $\ell(\lambda)=3$.

\footnotetext{
*Partially supported by NSF Grants DMS-1500838 and DMS-1953781.
} 
Definition 1. (Griffin [5]) Let $k \leqslant n$ be nonnegative integers and let $\lambda=\left(\lambda_{1} \geqslant \cdots \geqslant\right.$ $\left.\lambda_{s} \geqslant 0\right)$ be a partition of $k$ with $s$ parts. Write $\lambda^{\prime}=\left(\lambda_{1}^{\prime} \geqslant \cdots \geqslant \lambda_{n}^{\prime} \geqslant 0\right)$ for the conjugate partition of $\lambda$, padded with trailing zeros to have $n$ parts.

Let $I_{n, \lambda} \subseteq \mathbb{Q}\left[\mathbf{x}_{n}\right]$ be the ideal

$$
I_{n, \lambda}=\left\langle x_{1}^{s}, \ldots, x_{n}^{s}\right\rangle+\left\langle e_{d}(S): S \subseteq[n] \text { and } d>|S|-\lambda_{n}^{\prime}-\lambda_{n-1}^{\prime}-\cdots-\lambda_{n-|S|+1}^{\prime}\right\rangle
$$

and let $R_{n, \lambda}:=\mathbb{Q}\left[\mathbf{x}_{n}\right] / I_{n, \lambda}$ be the associated quotient ring ${ }^{1}$.

As an example, suppose $n=9$ and $\lambda=(3,2,2,0)$ so that $k=7$ and $s=4$. The conjugate partition $\lambda^{\prime}=\left(\lambda_{1}^{\prime}, \ldots, \lambda_{9}^{\prime}\right)$ is given by $(3,3,1,0,0,0,0,0,0)$. The ideal $I_{9, \lambda} \subseteq$ $\mathbb{Q}\left[\mathbf{x}_{9}\right]$ is generated by $x_{1}^{4}, \ldots, x_{9}^{4}$ together with the polynomials

$$
\begin{array}{llll}
e_{i}(S) & S \subseteq[9] & |S|=9 & i=9,8,7,6,5,4,3 \\
e_{j}(T) & T \subseteq[9] & |T|=8 & j=8,7,6,5 \\
e_{d}(U) & U \subseteq[9] & |U|=7 & d=7 .
\end{array}
$$

Griffin's rings $R_{n, \lambda}$ generalize several important classes of quotient rings in algebraic combinatorics.

- When $k=s=n$ and $\lambda=\left(1^{n}\right)$, the ideal $I_{n, \lambda}$ is generated by the $n$ elementary symmetric polynomials $e_{1}\left(\mathbf{x}_{n}\right), e_{2}\left(\mathbf{x}_{n}\right), \ldots, e_{n}\left(\mathbf{x}_{n}\right)$ in the full variable set $\left\{x_{1}, \ldots, x_{n}\right\}$ and $R_{n, \lambda}$ is the classical coinvariant ring

$$
R_{n}:=\mathbb{Q}\left[\mathbf{x}_{n}\right] /\left\langle e_{1}\left(\mathbf{x}_{n}\right), e_{2}\left(\mathbf{x}_{n}\right), \ldots, e_{n}\left(\mathbf{x}_{n}\right)\right\rangle
$$

attached to the symmetric group $\mathfrak{S}_{n}$. The ring $R_{n, \lambda}$ presents the cohomology of the complete flag variety of type $\mathrm{A}_{n-1}$; see [3] for the details.

- When $k=n$ and $\lambda \vdash n$ is arbitrary, the ring $R_{n, \lambda}$ is the Tanisaki quotient studied by Tanisaki [14] and Garsia-Procesi [4] which presents the cohomology of the Springer fiber $\mathcal{B}_{\lambda}$ attached to the partition $\lambda$.

- When $\lambda=\left(1^{k}, 0^{s-k}\right)$ has all parts $\leqslant 1$, the rings $R_{n, \lambda}$ were introduced by Haglund, Rhoades and Shimozono [8] to give a representation-theoretic model for the HaglundRemmel-Wilson Delta Conjecture [7]. Pawlowski-Rhoades proved that these rings present the cohomology of the moduli space of $n$-tuples of lines $\left(\ell_{1}, \ldots, \ell_{n}\right)$ in $\mathbb{C}^{s}$ such that the composite linear map

$$
\ell_{1} \oplus \cdots \oplus \ell_{n} \rightarrow \mathbb{C}^{s} \rightarrow \mathbb{C}^{k}
$$

given by addition $\left(v_{1}, \ldots, v_{n}\right) \mapsto v_{1}+\cdots+v_{n}$ followed by projection onto the first $k$ coordinates is a surjection [10].

\footnotetext{
${ }^{1}$ Our notation differs from that of Griffin [5]. He considers partitions to only have positive parts and includes $s \geqslant \ell(\lambda)$ as a parameter, so that his objects are denoted $I_{n, \lambda, s}$ and $R_{n, \lambda, s}$. Griffin uses the symbol $I_{n, \lambda}$ to denote the ideal $\left\langle e_{d}(S): S \subseteq[n]\right.$ and $\left.d>|S|-\lambda_{n}^{\prime}-\lambda_{n-1}^{\prime}-\cdots-\lambda_{n-|S|+1}^{\prime}\right\rangle$ and $R_{n, \lambda}$ to denote the corresponding quotient - this is the 'limit' as $s \rightarrow \infty$ of his $I_{n, \lambda, s}$ and $R_{n, \lambda, s}$.
} 
The symmetric group $\mathfrak{S}_{n}$ acts on $\mathbb{Q}\left[\mathbf{x}_{n}\right]$ by subscript permutation. The ideals $I_{n, \lambda}$ are graded and $\mathfrak{S}_{n}$-stable, so $R_{n, \lambda}$ is a graded $\mathfrak{S}_{n}$-module. Generalizing results from $[4,8]$, Griffin calculated [5] the graded $\mathfrak{S}_{n}$-isomorphism type of $R_{n, \lambda}$. It is an open problem to find a variety $X_{n, \lambda}$ whose cohomology is presented by $R_{n, \lambda}$, but Griffin proved that $R_{n, \lambda}$ is the coordinate ring of a scheme-theoretic intersection arising from rank varieties [5].

In this paper we study the rings $R_{n, \lambda}$ as graded $\mathbb{Q}$-vector spaces. In the special case $k=s=n$ and $\lambda=\left(1^{n}\right)$, the classical coinvariant ring $R_{n}$ has a number of interesting bases which are important for different reasons. Perhaps the simplest of these was discovered by E. Artin [1], who used Galois Theory to prove that the family of 'sub-staircase monomials'

$$
\left\{x_{1}^{c_{1}} x_{2}^{c_{2}} \cdots x_{n}^{c_{n}}: 0 \leqslant c_{i}<n-i\right\}
$$

descends to a basis for $R_{n}$. Extending earlier results of [4, 8], Griffin discovered the appropriate generalization of 'sub-staircase' to obtain a monomial basis of $R_{n, \lambda}$; his result is quoted in Theorem 4 below.

Our main goal in this paper is to describe the harmonic space of the quotient ring $R_{n, \lambda}$ and so derive a harmonic basis of this quotient ring. In order to motivate harmonic spaces and bases, we recall some technical issues that arise in the study of quotient rings.

Let $I \subseteq \mathbb{Q}\left[\mathbf{x}_{n}\right]$ be any homogeneous ideal with quotient ring $R=\mathbb{Q}\left[\mathbf{x}_{n}\right] / I$. In algebraic combinatorics, one is often interested in calculating algebraic invariants of $R$ such as its dimension or Hilbert series. A frequent impediment to computing these invariants is that, given $f \in \mathbb{Q}\left[\mathbf{x}_{n}\right]$, it can be difficult to decide whether $f+I=0$ in $R$. Harmonic spaces can be used to replace quotients with subspaces, circumventing this problem.

For $f=f\left(x_{1}, \ldots, x_{n}\right) \in \mathbb{Q}\left[\mathbf{x}_{n}\right]$, let $\partial f:=f\left(\partial / \partial x_{1}, \ldots, \partial / \partial x_{n}\right)$ be the differential operator on $\mathbb{Q}\left[\mathbf{x}_{n}\right]$ obtained by replacing each $x_{i}$ appearing in $f$ with the partial derivative $\partial / \partial x_{i}$. The ring $\mathbb{Q}\left[\mathbf{x}_{n}\right]$ acts on itself by

$$
f \odot g:=(\partial f)(g) \text { for all } f, g \in \mathbb{Q}\left[\mathbf{x}_{n}\right] .
$$

That is, the polynomial $f \odot g$ is obtained by first turning $f$ into a differential operator $\partial f$, and then applying $\partial f$ to $g$.

For $f, g \in \mathbb{Q}\left[\mathbf{x}_{n}\right]$, we define a number $\langle f, g\rangle \in \mathbb{Q}$ by

$$
\langle f, g\rangle:=\text { constant term of } f \odot g \text {. }
$$

Given two monomials $x_{1}^{a_{1}} \cdots x_{n}^{a_{n}}$ and $x_{1}^{b_{1}} \cdots x_{n}^{b_{n}}$, it follows directly that

$$
\left\langle x_{1}^{a_{1}} \cdots x_{n}^{a_{n}}, x_{1}^{b_{1}} \cdots x_{n}^{b_{n}}\right\rangle= \begin{cases}a_{1} ! \cdots a_{n} ! & \text { if } a_{i}=b_{i} \text { for all } i \\ 0 & \text { otherwise }\end{cases}
$$

so that $\langle-,-\rangle$ is an inner product on $\mathbb{Q}\left[\mathbf{x}_{n}\right]$ for which the degree grading $\mathbb{Q}\left[\mathbf{x}_{n}\right]=$ $\bigoplus_{d \geqslant 0} \mathbb{Q}\left[\mathbf{x}_{n}\right]_{d}$ is an orthogonal decomposition.

For a homogeneous ideal $I \subseteq \mathbb{Q}\left[\mathbf{x}_{n}\right]$, the harmonic space $V$ of $I$ is the graded subspace of $\mathbb{Q}\left[\mathbf{x}_{n}\right]$ given by

$$
V=I^{\perp}=\left\{g \in \mathbb{Q}\left[\mathbf{x}_{n}\right]:\langle f, g\rangle=0 \text { for all } f \in I\right\} .
$$


Writing $R=\mathbb{Q}\left[\mathbf{x}_{n}\right] / I$, standard results of linear algebra imply that $\mathbb{Q}\left[\mathbf{x}_{n}\right]=V \oplus I$ so that any vector space basis for $V$ projects onto a basis of $R$. Any basis of $V$ (and its image basis in $R$ ) is called a harmonic basis. If the ideal $I$ is $\mathfrak{S}_{n}$-invariant, the $\mathfrak{S}_{n}$-invariance of the inner product $\langle-,-\rangle$ furnishes an isomorphism of graded $\mathfrak{S}_{n}$-modules $R \cong V$. The harmonic space $V$ therefore permits the study of the quotient ring $R$ without the computational issues inherent in cosets.

Definition 2. Let $V_{n, \lambda} \subseteq \mathbb{Q}\left[\mathbf{x}_{n}\right]$ be the harmonic space of $I_{n, \lambda}$.

We have an isomorphism of graded $\mathfrak{S}_{n}$-modules $R_{n, \lambda} \cong V_{n, \lambda}$ and any basis for $V_{n, \lambda}$ descends to a basis for $R_{n, \lambda}$. In the classical case $k=s=n$ and $\lambda=(1, \ldots, 1)$ so that $R_{n, \lambda}=R_{n}$, the harmonic space $V_{n, \lambda}$ has the following description.

Recall that the Vandermonde determinant $\delta_{n} \in \mathbb{Q}\left[\mathbf{x}_{n}\right]$ is the polynomial

$$
\delta_{n}:=\prod_{1 \leqslant i<j \leqslant n}\left(x_{i}-x_{j}\right) .
$$

The harmonic space $V_{n} \subseteq \mathbb{Q}\left[\mathbf{x}_{n}\right]$ corresponding to $R_{n}$ is generated by $\delta_{n}$ as a $\mathbb{Q}\left[\mathbf{x}_{n}\right]$ module. More explicitly, the space $V_{n}$ is the smallest subspace of $\mathbb{Q}\left[\mathbf{x}_{n}\right]$ containing $\delta_{n}$ which is closed under the partial derivatives $\partial / \partial x_{1}, \ldots, \partial / \partial x_{n}$. A harmonic basis of $R_{n}$ is given by applying sub-staircase monomials (as differential operators) to $\delta_{n}$ :

$$
\left\{\left(x_{1}^{c_{1}} \cdots x_{n}^{c_{n}}\right) \odot \delta_{n}: 0 \leqslant c_{i} \leqslant n-i\right\} .
$$

In the Springer fiber case $k=n$ with $\lambda$ arbitrary, the harmonic space $V_{n, \lambda}$ was described by N. Bergeron and Garsia [2] using 'partial Vandermonde' polynomials.

In order to describe our results, we need one more definition. Given $k \leqslant n$ and a partition $\lambda=\left(\lambda_{1} \geqslant \cdots \geqslant \lambda_{s}\right)$ of $k$, let $\mathcal{O P}_{n, \lambda}$ be the collection of length $s$ sequences $\sigma=\left(B_{1}|\cdots| B_{s}\right)$ of subsets of $[n]$ such that

- we have a disjoint union decomposition $[n]=B_{1} \sqcup \cdots \sqcup B_{s}$, and

- the set $B_{i}$ has at least $\lambda_{i}$ elements.

We refer to elements $\sigma \in \mathcal{O P}_{n, \lambda}$ as ordered set partitions, even though some of the sets $B_{i}$ may be empty when the partition $\lambda$ has trailing zeros. When $k=s=n$ and $\lambda=\left(1^{n}\right)$, we have an identification $\mathcal{O P}_{n, \lambda}=\mathfrak{S}_{n}$ of ordered set partitions and permutations.

- We generalize work of Rhoades-Wilson [12] to define a coinversion statistic $\operatorname{coinv}(\sigma)$ on $\mathcal{O} \mathcal{P}_{n, \lambda}$ and an extension code $(\sigma)$ of the Lehmer code of a permutation to $\mathcal{O} \mathcal{P}_{n, \lambda}$ (Section 3). We show that the map $\sigma \mapsto \operatorname{code}(\sigma)$ bijects $\mathcal{O P} \mathcal{P}_{n, \lambda}$ with a family of sequences $\mathcal{C}_{n, \lambda}$ determined by $n$ and $\lambda$ (Theorem 8 ).

- We show that the Hilbert series of $R_{n, \lambda}$ is the generating function of the statistic coinv on $\mathcal{O P}_{n, \lambda}$ (Corollary 17). 
- We describe a generating set for the harmonic space $V_{n, \lambda}$ as a $\mathbb{Q}\left[\mathbf{x}_{n}\right]$-module (Theorem 15) and give an explicit harmonic basis $\left\{\delta_{\sigma}: \sigma \in \mathcal{O} \mathcal{P}_{n, \lambda}\right\}$ of $R_{n, \lambda}$ indexed by ordered set partitions in $\mathcal{O P}_{n, \lambda}$ (Theorem 16).

- We show that the lexicographical leading monomials of the harmonic polynomials in $V_{n, \lambda}$ are precisely those with exponent sequences lying in $\mathcal{C}_{n, \lambda}$ (Theorem 20).

The rest of the paper is organized as follows. In Section $\mathbf{2}$ we give background on partitions, tableaux, and ordered set partitions. In Section 3 we describe our new coinversion statistic on $\mathcal{O P}_{n, \lambda}$ as well as its associated coinversion code. We use an insertion algorithm to describe the possible coinversion codes of elements of $\mathcal{O} \mathcal{P}_{n, \lambda}$. In Section 4 we study the harmonic space $V_{n, \lambda}$ and, in particular, give a harmonic basis for $V_{n, \lambda}$ (or $R_{n, \lambda}$ ) indexed by $\mathcal{O P}_{n, \lambda}$. We also prove a conjecture of $\mathrm{A}$. T. Wilson (personal communication) by showing that a certain family $R_{n, k, s}$ of graded rings coincides with a special case of Griffin's rings $R_{n, \lambda}$. We close in Section 5 by proposing a connection between this work and superspace.

\section{Background}

\subsection{Partitions and tableaux}

Given $k \geqslant 0$, a partition of $n$ is a weakly decreasing sequence $\lambda=\left(\lambda_{1} \geqslant \cdots \geqslant \lambda_{s}\right)$ of nonnegative integers satisfying $\lambda_{1}+\cdots+\lambda_{s}=k$. In particular, we allow trailing zeros in our partitions. Let $\ell(\lambda)$ denote the number of nonzero parts of a partition $\lambda$. We use the notation $\lambda \vdash k$ to indicate that $\lambda$ is a partition of $k$.

The Young diagram of a partition $\lambda$ consists of $\lambda_{i}$ left-justified boxes in row $i$. For example, the Young diagram of $(4,2,1) \vdash 7$ is shown below.

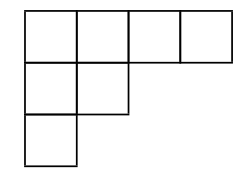

Observe that trailing zeros have no effect on Young diagrams, so this would also be the Young diagram of the partition $(4,2,1,0,0)$. The conjugate $\lambda^{\prime}$ of a partition $\lambda$ is obtained by reflecting its Young diagram across the main diagonal; we have $\lambda^{\prime}=(3,2,1,1)$ in this case.

Let $\lambda$ be a partition. A tableau $T$ of shape $\lambda$ is a filling $T: \lambda \rightarrow\{1,2, \ldots\}$ of the boxes of $\lambda$ with positive integers. A tableau $T$ is column strict if its entries strictly increase going down columns and injective if its entries are distinct. We write $\operatorname{Inj}(\lambda, \leqslant n)$ for the family of injective and column strict tableaux of shape $\lambda$ whose entries are bounded above by $n$. An example tableau in $\operatorname{Inj}((4,2,1), \leqslant 9)$ is shown below; observe that the numbers 7 and 8 do not appear in this tableau.

$$
\begin{array}{|l|l|l|l|}
\hline 2 & 1 & 3 & 9 \\
\cline { 1 - 2 } 5 & 4 & \multicolumn{2}{|c}{} \\
\cline { 1 - 2 } 6 & \multicolumn{3}{|c}{} \\
\cline { 1 - 2 } & &
\end{array}
$$




\subsection{Ordered set partitions}

A (weak) ordered set partition of $[n]$ is a sequence $\sigma=\left(B_{1}|\cdots| B_{s}\right)$ of (possibly empty) subsets of $[n]$ such that we have a disjoint union decomposition $[n]=B_{1} \sqcup \cdots \sqcup B_{s}$. We say that $\sigma$ has $s$ blocks. As an example,

$$
\sigma=(1,3,5,9|6,7,8,10,14| 2,12,15|4,13| \varnothing \mid 11,16)
$$

is an ordered set partition of [16] with 6 blocks.

Let $\lambda=\left(\lambda_{1} \geqslant \cdots \geqslant \lambda_{s}\right)$ be a partition. As described in the introduction, we write $\mathcal{O P} \mathcal{P}_{n, \lambda}$ for the family of all ordered set partitions $\sigma=\left(B_{1}|\cdots| B_{s}\right)$ of $[n]$ with $s$ blocks such that $B_{i}$ has at least $\lambda_{i}$ elements, for all $1 \leqslant i \leqslant s$. If $\lambda=(3,3,2,2,0,0)$, the ordered set partition $\sigma$ above lies in $\mathcal{O} \mathcal{P}_{16, \lambda}$.

It will be convenient to visualize elements of $\mathcal{O} \mathcal{P}_{n, \lambda}$ in terms of the following container diagrams. Given $\lambda=\left(\lambda_{1} \geqslant \cdots \geqslant \lambda_{s}\right)$, we first draw (from left to right) $s$ columns of top-justified boxes of height $\lambda_{i}$. (These boxes are called the container.) For $\sigma=\left(B_{1} \mid\right.$ $\left.\cdots \mid B_{s}\right) \in \mathcal{O} \mathcal{P}_{n, \lambda}$, we fill the $i^{\text {th }}$ column with the entries of $B_{i}$, increasing from bottom to top. Our example ordered set partition $\sigma \in \mathcal{O P}_{16,(3,3,2,2,0,0)}$ has the following container diagram, with column numberings corresponding to block indices.

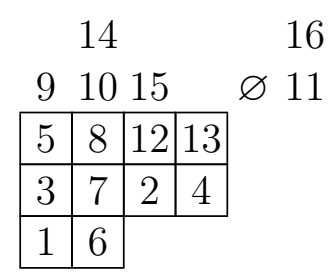

$$
\begin{array}{llllll}
1 & 2 & 3 & 4 & 5 & 6
\end{array}
$$

Empty blocks in ordered set partitions give rise to empty columns in container diagrams. The container diagram above has a single empty column, decorated with the placeholder $\varnothing$. The condition $\sigma \in \mathcal{O P}_{n, \lambda}$ corresponds to the container of boxes being completely filled with numbers. The numbers appearing outsider of the container $(9,10,11,14,15$, and 16 in our example) are called floating.

\section{Coinversion codes for $\mathcal{O P}_{n, \lambda}$}

\subsection{Coinversions in ordered set partitions}

One variant of the Lehmer code of a permutation $\pi=\pi_{1} \ldots \pi_{n} \in \mathfrak{S}_{n}$ is given by the sequence $\left(c_{1}, \ldots, c_{n}\right)$ where

$$
c_{i}=\left|\left\{i<j: \pi_{i}<\pi_{j}\right\}\right| .
$$

The sum of this sequence $c_{1}+\cdots+c_{n}$ counts the total number of coinversions (i.e. noninversions) of $\pi$. We extend this definition from permutations to ordered set partitions as follows. 
Let $\lambda=\left(\lambda_{1} \geqslant \cdots \geqslant \lambda_{s}\right) \vdash k$ be a partition, let $n \geqslant k$, and let $\sigma \in \mathcal{O P}_{n, \lambda}$. We think of $\sigma$ in terms of its container diagram. For $1 \leqslant i<j \leqslant n$, we say that $(i, j)$ is a coinversion of $\sigma$ when one of the following three conditions hold:

- $i$ is not floating, $j$ is to the right of $i$ in $\sigma, i$ and $j$ are in the same row of $\sigma$, and $i<j$,

- $i$ is not floating, $j$ is to the left of $i$ in $\sigma, j$ is one row below $i$ in $\sigma$, and $i<j$, or

- $i$ is floating, $j$ is to the right of $i$ in $\sigma, j$ is at the top of its container, and $i<j$.

The first two conditions may be depicted schematically as

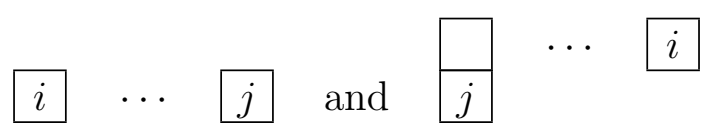

where $i$ and $j$ are in adjacent rows in the diagram on the right. The last condition may be depicted

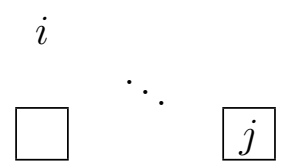

where $j$ is at the top of the container and there is an arbitrary positive number of rows separating $i$ and $j$.

Remark 3. The conditions defining coinversions for non-floating indices are the same as those used to define the statistic dinv which arises in the Haglund-Haiman-Loehr monomial expansion of the modified Macdonald polynomials [6].

For $1 \leqslant i \leqslant n$ we define a number $c_{i} \geqslant 0$ by

$$
c_{i}:= \begin{cases}\mid\{i<j:(i, j) \text { is a coinversion of } \sigma\} \mid & \text { if } i \text { is not floating } \\ \mid\{i<j:(i, j) \text { is a coinversion of } \sigma\} \mid+(p-1) & \text { if } i \text { is floating in the } \\ & p^{t h} \text { block of } \sigma .\end{cases}
$$

The coinversion code of $\sigma$ is given by

$$
\operatorname{code}(\sigma):=\left(c_{1}, \ldots, c_{n}\right)
$$

and the coinversion number of $\sigma$ is given by

$$
\operatorname{coinv}(\sigma):=c_{1}+\cdots+c_{n} .
$$

Rhoades and Wilson [12] defined $\operatorname{code}(\sigma)$ in the special case where $\lambda=\left(1^{k}\right)$.

As an example of these concepts, consider the ordered set partition $\sigma \in \mathcal{O P}_{16,(3,3,2,2,0,0)}$ appearing in (11). Let $\left(c_{1}, \ldots, c_{16}\right)$ be the sequence code $(\sigma)$. The entry 2 forms coinversions with 4 and 6 , so that $c_{2}=2$. The entry 10 is floating in column 2 , and forms coinversions with 12 and 13 so that $c_{10}=2+(2-1)=3$. We have

$$
\operatorname{code}(\sigma)=\left(c_{1}, \ldots, c_{16}\right)=(1,2,2,1,3,0,0,2,2,3,5,1,0,1,2,5) .
$$

Adding this sequence yields $\operatorname{coinv}(\sigma)=30$. 


\subsection{The family of sequences $\mathcal{C}_{n, \lambda}$}

The map $\sigma \mapsto \operatorname{code}(\sigma)$ assigning $\sigma \in \mathcal{O P}_{n, \lambda}$ to its coinversion code will turn out to be an injection. In order to describe the image of this map, we recall that a shuffle of two sequences $\left(a_{1}, \ldots, a_{p}\right)$ and $\left(b_{1}, \ldots, b_{q}\right)$ is an interleaving $\left(c_{1}, \ldots, c_{p+q}\right)$ of these sequences which preserves the relative order of the $a$ 's and the $b$ 's. A shuffle of any finite number of sequences may be defined analogously (or inductively).

Let $k \leqslant n$ be positive integers, let $\lambda=\left(\lambda_{1} \geqslant \cdots \geqslant \lambda_{s}\right) \vdash k$ be a partition with $s$ nonnegative parts, and write the conjugate of $\lambda$ as $\left(\lambda_{1}^{\prime} \geqslant \cdots \geqslant \lambda_{k}^{\prime}\right)$. We define $\mathcal{C}_{n, \lambda}$ to be the family of length $n$ sequences $\left(c_{1}, \ldots, c_{n}\right)$ of nonnegative integers which are componentwise $\leqslant$ some shuffle of the $k+1$ (possibly empty) sequences

$$
\left(\lambda_{1}^{\prime}-1, \lambda_{1}^{\prime}-2, \ldots, 1,0\right), \ldots,\left(\lambda_{k}^{\prime}-1, \lambda_{k}^{\prime}-2, \ldots, 1,0\right), \text { and }(s-1, s-1, \ldots, s-1),
$$

where the final sequence has $n-k$ copies of $s-1$.

Continuing our running example of $n=16$ and $\lambda=(3,3,2,2,0,0) \vdash 8$, the nonzero parts of $\lambda^{\prime}$ are $(4,4,2)$ so that $\mathcal{C}_{n, \lambda}$ consists of all length 16 sequences $\left(c_{1}, \ldots, c_{16}\right)$ of nonnegative integers which are componentwise $\leqslant$ some shuffle of the sequences

$$
(3,2,1,0),(3,2,1,0),(1,0) \text {, and }(5,5,5,5,5,5) \text {. }
$$

The sequence family $\mathcal{C}_{n, \lambda}$ was introduced by Haglund-Rhoades-Shimozono [8] in the case $\lambda_{1} \leqslant 1$ and by Griffin [5] for general $\lambda$. Griffin proved that the monomials in $\mathbb{Q}\left[\mathbf{x}_{n}\right]$ whose exponent sequences lie in $\mathcal{C}_{n, \lambda}$ descend to a basis of the ring $R_{n, \lambda}$.

Theorem 4. (Griffin [5]) Let $k \leqslant n$ be positive integers and let $\lambda=\left(\lambda_{1} \geqslant \cdots \geqslant \lambda_{s}\right) \vdash k$. The set of monomials

$$
\left\{x_{1}^{c_{1}} \cdots x_{n}^{c_{n}}:\left(c_{1}, \ldots, c_{n}\right) \in \mathcal{C}_{n, \lambda}\right\}
$$

descends to a vector space basis of $R_{n, \lambda}$.

We will prove that code is a bijection from $\mathcal{O P}_{n, \lambda}$ to $\mathcal{C}_{n, \lambda}$. As a first step, we show that $\operatorname{code}(\sigma) \in \mathcal{C}_{n, \lambda}$ for any $\sigma \in \mathcal{O P}_{n, \lambda}$.

Lemma 5. Let $k \leqslant n$ be positive integers and let $\lambda=\left(\lambda_{1} \geqslant \cdots \geqslant \lambda_{s}\right) \vdash k$ be a partition of $k$. For any $\sigma \in \mathcal{O P}_{n, \lambda}$ we have $\operatorname{code}(\sigma) \in \mathcal{C}_{n, \lambda}$.

Proof. The $i^{\text {th }}$ row from the top of the container of $\sigma$ contains $\lambda_{i}^{\prime}$ boxes. It follows from the definition of coinversions that the $j^{\text {th }}$ smallest entry in this row forms at most $\lambda_{i}^{\prime}-j$ coinversions with other entries of $\sigma$. Furthermore, if $t$ is any floating entry of $\sigma$, then $c_{t} \leqslant s-1$ by construction. The entries in the $\lambda_{1}$ rows of $\sigma$, together with the $n-k$ floating entries, define a shuffle $\left(c_{1}^{\prime}, \ldots, c_{n}^{\prime}\right)$ of the sequences

$$
\left(\lambda_{1}^{\prime}-1, \lambda_{1}^{\prime}-2, \ldots, 1,0\right), \ldots,\left(\lambda_{k}^{\prime}-1, \lambda_{k}^{\prime}-2, \ldots, 1,0\right), \text { and }(\overbrace{s-1, s-1, \ldots, s-1}^{n-k}) \text {, }
$$

such that we have the componentwise inequality $\operatorname{code}(\sigma) \leqslant\left(c_{1}^{\prime}, \ldots, c_{n}^{\prime}\right)$. 
To see how this works, suppose $\sigma$ is as in (11):

$$
\sigma=\begin{array}{|l|l|l|l|}
\multicolumn{1}{c}{14} & \\
9 & 10 & 15 \\
\hline 5 & 8 & 12 & 13 \\
\hline 3 & 7 & 2 & 4 \\
\hline 1 & 6 & \multicolumn{1}{|c}{} \\
\end{array}
$$

We use the container diagram of $\sigma$ to form a shuffle $\left(c_{1}^{\prime}, \ldots, c_{16}^{\prime}\right)$ of the sequences

$$
\left(3^{\bullet}, 2^{\bullet}, 1^{\bullet}, 0^{\bullet}\right),\left(3^{\circ}, 2^{\circ}, 1^{\circ}, 0^{\circ}\right),\left(1^{\square}, 0^{\square}\right) \text {, and }(5,5,5,5,5,5) \text {. }
$$

Here we label our sequences with decorations $(\bullet, \circ, \square$, and unadorned) so that we can distinguish them when we perform our shuffle. The shuffle $\left(c_{1}^{\prime}, \ldots, c_{16}^{\prime}\right)$ corresponding to $\sigma$ is:

$$
\left(c_{1}^{\prime}, \ldots c_{16}^{\prime}\right):=\left(1^{\square}, 3^{\circ}, 2^{\circ}, 1^{\circ}, 3^{\bullet}, 0^{\square}, 0^{\circ}, 2^{\bullet}, 5,5,5,1^{\bullet}, 0^{\bullet}, 5,5,5\right) .
$$

The positions of the $\bullet$ entries are given by top row of the container of $\sigma(5,8,12$, and 13$)$. The positions of the $\circ$ entries $(2,4,3$, and 7$)$ are the middle row of the container and the positions of the $\square$ entries ( 1 and 6 ) are the bottom row of the container. The unadorned entries (in positions 9, 10,11, 14, 15, and 16) are the floating numbers. The reader can verify the componentwise inequality $\operatorname{code}(\sigma) \leqslant\left(c_{1}^{\prime}, \ldots, c_{n}^{\prime}\right)$.

The shuffle $\left(c_{1}^{\prime}, \ldots, c_{n}^{\prime}\right)$ constructed in the proof of Lemma 5 will be important in Section 4 , so we give it a name.

Definition 6. Let $\sigma \in \mathcal{O P}_{n, \lambda}$ for some partition $\lambda=\left(\lambda_{1} \geqslant \cdots \geqslant \lambda_{s}\right)$ of $k$. The shuffle $\left(c_{1}^{\prime}, \ldots, c_{n}^{\prime}\right)$ of the sequences of the sequences

$$
\left(\lambda_{1}^{\prime}-1, \lambda_{1}^{\prime}-2, \ldots, 1,0\right), \ldots,\left(\lambda_{k}^{\prime}-1, \lambda_{k}^{\prime}-2, \ldots, 1,0\right), \text { and }(\overbrace{s-1, s-1, \ldots, s-1}^{n-k})
$$

obtained by placing $\left(\lambda_{i}^{\prime}-1, \lambda_{i}^{\prime}-2, \ldots, 1,0\right)$ into the positions contained in the $i^{\text {th }}$ row from the top of the container of $\sigma$, and placing $s-1$ into all floating positions of $\sigma$, will be referred to as maxcode $(\sigma)$.

For example, if $\sigma=(1,3,5,9|6,7,8,10,14| 2,12,15|4,13| \varnothing \mid 11,16) \in$ $\mathcal{O P} \mathcal{P}_{16,(3,3,2,2,0,0)}$ is the ordered set partition in the proof of Lemma 5 then

$$
\operatorname{maxcode}(\sigma)=(1,3,2,1,3,0,0,2,5,5,5,1,0,5,5,5) .
$$

The proof of Lemma 5 gives the following result immediately.

Lemma 7. For any $\sigma \in \mathcal{O P}_{n, \lambda}$ we have the componentwise inequality

$$
\operatorname{code}(\sigma) \leqslant \operatorname{maxcode}(\sigma)
$$


By Lemma 5, we have a well-defined map

$$
\text { code }: \mathcal{O P} \mathcal{P}_{n, \lambda} \rightarrow \mathcal{C}_{n, \lambda}
$$

which sends $\sigma \in \mathcal{O P}_{n, \lambda}$ to its coinversion code $\operatorname{code}(\sigma)=\left(c_{1}, \ldots, c_{n}\right)$. Our first main result states that this map is a bijection.

Theorem 8. Let $k \leqslant n$ be positive integers and let $\lambda=\left(\lambda_{1} \geqslant \cdots \geqslant \lambda_{s}\right) \vdash k$ be a partition of $k$. The map code : $\mathcal{O P}_{n, \lambda} \rightarrow \mathcal{C}_{n, \lambda}$ is a bijection.

Proof. In order to prove that code is a bijection, we construct its inverse $\mathcal{C}_{n, \lambda} \rightarrow \mathcal{O P}_{n, \lambda}$. Given $\left(c_{1}, \ldots, c_{n}\right) \in \mathcal{C}_{n, \lambda}$, we define $\iota\left(c_{1}, \ldots, c_{n}\right) \in \mathcal{O P}_{n, \lambda}$ by the following insertion algorithm.

The element $\iota\left(c_{1}, \ldots, c_{n}\right) \in \mathcal{O P}_{n, \lambda}$ will be constructed by starting with an empty container of shape $\lambda$ and inserting the numbers $1,2, \ldots, n$ (in that order) to yield an element of $\mathcal{O P} \mathcal{P}_{n, \lambda}$. To describe what happens at a typical step of this insertion process, consider an ordered set partition $\left(B_{1}|\cdots| B_{s}\right)$ with $s$ blocks. We place the blocks $B_{1}, \ldots, B_{s}$ in the container diagram corresponding to $\lambda$, from left to right. For example, if $\lambda=(3,3,2,2,0,0)$ and

$$
\left(B_{1}|\cdots| B_{s}\right)=(4|2,3,6| 1|\varnothing| \varnothing \mid 5)
$$

our diagram is shown below. In particular, the first, third, and fourth container columns from the left remain unfilled.

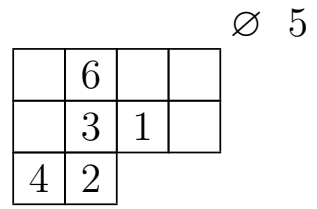

$$
\begin{array}{llllll}
1 & 3 & 2 & 0 & 4 & 5
\end{array}
$$

We label the blocks of $\left(B_{1}|\cdots| B_{s}\right)$ (or equivalently the columns of its container diagram) with the $s$ distinct coinversion labels $0,1,2, \ldots, s-1$ according to the following rules

1. any unfilled container column receives a smaller coinversion label than any filled container column,

2. the coinversion labels of the filled container columns increase from left to right,

3. given two unfilled container columns with different numbers of empty boxes, the column with more empty boxes has a smaller coinversion label,

4. the coinversion labels of unfilled container columns with the same numbers of empty boxes increase from right to left. 
The unique labeling satisfying (1) - (4) may be obtained by first labeling the $a$ columns which contain no empty boxes (including those columns corresponding to empty parts of $\lambda$ ) from right to left with $s-1, s-2, \ldots, s-a$, then labeling the $b$ columns which contain a single empty box from left to right with $s-a-1, s-a-2, \ldots, s-a-b$, then labeling the $c$ columns which contain two empty boxes from left to right with $s-a-b-1, s-$ $a-b-2, \ldots, s-a-b-c$, and so on. (Notice that coinversion labels are assigned 'in the opposite direction' when labeling filled and unfilled columns.) The coinversion labels are displayed below the columns of the container diagram.

The element $\iota\left(c_{1}, \ldots, c_{n}\right) \in \mathcal{O P}_{n, \lambda}$ is defined as follows. Starting with the 'empty' container diagram corresponding to $(\varnothing|\cdots| \varnothing)$, for each $i=1,2, \ldots, n$, we insert $i$ into the unique column with coinversion label $c_{i}$ (updating the coinversion labels as we go).

The map $\iota$ is best understood with an example. If $\lambda=(3,3,2,2,0,0)$ as above (so that $s=6$ ) and $n=16$, we have

$$
\left(c_{1}, \ldots, c_{16}\right)=(1,2,2,1,3,0,0,2,2,3,5,1,0,1,2,5) \in \mathcal{C}_{n, \lambda} .
$$

The insertion procedure defining $\iota\left(c_{1}, \ldots, c_{16}\right)$ proceeds as follows.

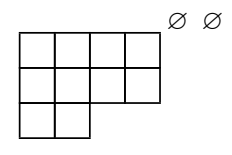

$\begin{array}{llllll}1 & 0 & 3 & 2 & 4 & 5\end{array}$

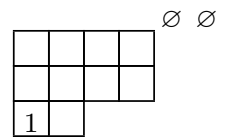

$\begin{array}{llllll}3 & 0 & 2 & 1 & 4 & 5\end{array}$

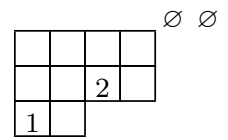

$\begin{array}{llllll}2 & 0 & 3 & 1 & 4 & 5\end{array}$

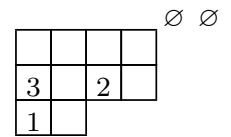

$\begin{array}{llllll}3 & 0 & 2 & 1 & 4 & 5\end{array}$

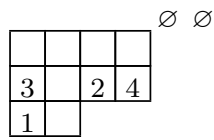

$\begin{array}{llllll}3 & 0 & 2 & 1 & 4 & 5\end{array}$

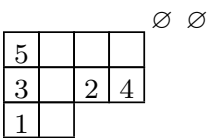

$\begin{array}{llllll}3 & 0 & 2 & 1 & 4 & 5\end{array}$

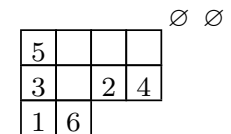

$\begin{array}{llllll}3 & 0 & 2 & 1 & 4 & 5\end{array}$

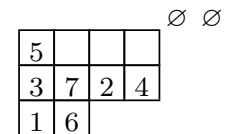

$\begin{array}{llllll}3 & 2 & 1 & 0 & 4 & 5\end{array}$

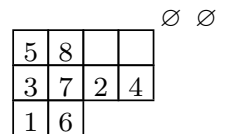

$\begin{array}{llllll}2 & 3 & 1 & 0 & 4 & 5\end{array}$

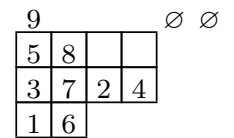

$\begin{array}{llllll}2 & 3 & 1 & 0 & 4 & 5\end{array}$

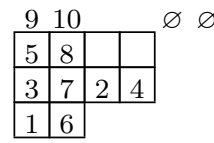

$\begin{array}{llllll}2 & 3 & 1 & 0 & 4 & 5\end{array}$

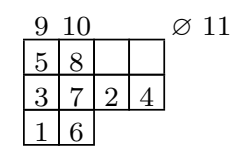

$\begin{array}{llllll}2 & 3 & 1 & 0 & 4 & 5\end{array}$

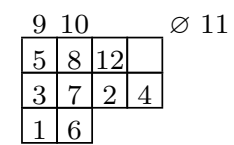

$\begin{array}{llllll}1 & 2 & 3 & 0 & 4 & 5\end{array}$

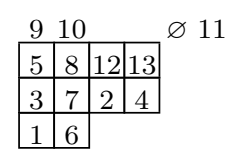

$\begin{array}{llllll}0 & 1 & 2 & 3 & 4 & 5\end{array}$

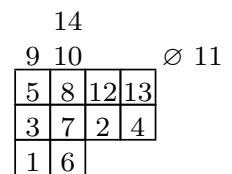

$\begin{array}{llllll}0 & 1 & 2 & 3 & 4 & 5\end{array}$

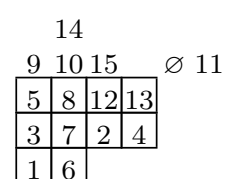

$\begin{array}{lllllll}0 & 1 & 2 & 3 & 4 & 5\end{array}$

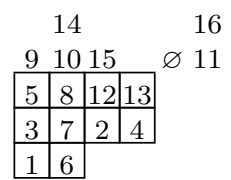

$\begin{array}{llllll}0 & 1 & 2 & 3 & 4 & 5\end{array}$

We conclude that $\iota\left(c_{1}, \ldots, c_{16}\right)$ is the element $\sigma \in \mathcal{O} \mathcal{P}_{16, \lambda}$ displayed in (11).

In order to verify that the map $\iota: \mathcal{C}_{n, \lambda} \rightarrow \mathcal{O} \mathcal{P}_{n, \lambda}$ is well-defined, we must show that the insertion procedure defining $\iota$ always fills every box in the container corresponding to $\lambda$. To do this, we induct on $n$.

Recall that $\ell(\lambda)$ is the number of nonzero parts in the partition $\lambda=\left(\lambda_{1} \geqslant \cdots \geqslant\right.$ $\left.\lambda_{s}\right)$ of $k$. For $1 \leqslant i \leqslant \ell(\lambda)$, let $\lambda^{(i)}$ be the partition obtained by sorting the sequence $\left(\lambda_{1}, \ldots, \lambda_{i}-1, \ldots \lambda_{s}\right)$ into weakly decreasing order. The set $\mathcal{C}_{n, \lambda}$ satisfies the following disjoint union decomposition based on the first entry of its sequences. 


$$
\begin{aligned}
\mathcal{C}_{n, \lambda}=\bigsqcup_{i=1}^{\ell(\lambda)}\left\{\left(i-1, c_{2}, \ldots, c_{n}\right):\left(c_{2}, \ldots, c_{n}\right) \in \mathcal{C}_{n-1, \lambda(i)}\right\} \sqcup \\
\qquad \bigsqcup_{j=\ell(\lambda)+1}^{s}\left\{\left(j-1, c_{2}, \ldots, c_{n}\right):\left(c_{2}, \ldots, c_{n}\right) \in \mathcal{C}_{n-1, \lambda}\right\}
\end{aligned}
$$

Equation (18) is equivalent to a result of Griffin [5, Lem. 3.8].

Given $\left(c_{1}, c_{2}, \ldots, c_{n}\right) \in \mathcal{C}_{n, \lambda}$, the algorithm $\iota$ starts by placing 1 in the column with coinversion label $c_{1}$. If $c_{1}<\ell(\lambda)$, the entry 1 fills a box in the container of $\lambda$, and the columns formed by the remaining container boxes (as well as their coinversion labels) rearrange to give the container corresponding to $\lambda^{\left(c_{1}+1\right)}$. If $c_{1} \geqslant \ell(\lambda)$, the entry 1 is floating, and the container remains unchanged. Equation (18) and induction on $n$ guarantee that the algorithm $\iota$ fills the container of $\lambda$, so that $\iota: \mathcal{C}_{n, \lambda} \rightarrow \mathcal{O P}_{n, \lambda}$ is well-defined. It is routine to check that the maps code and $\iota$ are mutually inverse.

\section{The harmonic space $V_{n, \lambda}$}

Throughout this section, we fix $k \leqslant n$ and let $\lambda=\left(\lambda_{1} \geqslant \cdots \geqslant \lambda_{s} \geqslant 0\right)$ be a partition of $k$ (with trailing zeros allowed). We write $\lambda^{\prime}$ for the partition conjugate to $\lambda$.

\subsection{Injective tableaux and their polynomials}

Let $T \in \operatorname{Inj}(\lambda, \leqslant n)$ be an injective tableau of shape $\lambda$ with entries $\leqslant n$. We introduce the monomial $\mathbf{x}(T)=x_{1}^{a_{1}} \cdots x_{n}^{a_{n}}$ where

$$
a_{i}= \begin{cases}b & \text { if } i \text { appears in } T \text { with } b \text { boxes directly below it, } \\ s-1 & \text { if } i \text { does not appear in } T .\end{cases}
$$

As an example, if $\lambda=(3,3,1,0,0)$ (so that $s=5)$ and

$$
T=\begin{array}{|l|l|l|}
\hline 2 & 1 & 3 \\
\hline 5 & 4 & 9 \\
\hline 6 &
\end{array}
$$

we have

$$
\mathbf{x}(T)=\left(x_{2}^{2} x_{5}^{1} x_{6}^{0}\right) \times\left(x_{1}^{1} x_{4}^{0}\right) \times\left(x_{3}^{1} x_{9}^{0}\right) \times\left(x_{7}^{4} x_{8}^{4}\right)=x_{1}^{1} x_{2}^{2} x_{3}^{1} x_{4}^{0} x_{5}^{1} x_{6}^{0} x_{7}^{4} x_{8}^{4} x_{9}^{0} .
$$

Given a tableau $T \in \operatorname{Inj}(\lambda, \leqslant n)$, we let $C_{T} \subseteq \mathfrak{S}_{n}$ be the parabolic subgroup of permutations $w \in \mathfrak{S}_{n}$ which stabilize the columns of $T$ and satisfy $w(i)=i$ for any $1 \leqslant$ $i \leqslant n$ which does not appear in $T$. In our case, we have $C_{T}=\mathfrak{S}_{\{2,5,6\}} \times \mathfrak{S}_{\{1,4\}} \times \mathfrak{S}_{\{3,9\}} \subseteq \mathfrak{S}_{9}$. We also define the group algebra element $\varepsilon_{T} \in \mathbb{Q}\left[\mathfrak{S}_{n}\right]$ by

$$
\varepsilon_{T}:=\sum_{w \in C_{T}} \operatorname{sign}(w) \cdot w
$$


Definition 9. Let $T \in \operatorname{Inj}(\lambda, \leqslant n)$. The polynomial $\delta_{T} \in \mathbb{Q}\left[\mathbf{x}_{n}\right]$ is

$$
\delta_{T}:=\varepsilon_{T} \cdot \mathbf{x}(T)
$$

the image of $\mathbf{x}(T)$ under the group algebra element $\varepsilon_{T}$.

The notation $\delta_{T}$ is justified as follows. If $T$ has columns $C_{1}, \ldots, C_{r}$, then $\delta_{T}$ factors as

$$
\delta_{T}=\delta_{C_{1}} \cdots \delta_{C_{r}} \times \prod_{\substack{1 \leqslant i \leqslant n \\ i \text { not appearing in } T}} x_{i}^{s-1}
$$

where $\delta_{C_{j}}$ is the Vandermonde in the set of variables whose indices appear in $C_{j}$. In our example we have

$$
\begin{aligned}
\delta_{T} & =\delta_{\{2,5,6\}} \times \delta_{\{1,4\}} \times \delta_{\{3,9\}} \times x_{7}^{4} x_{8}^{4} \\
& =\left(x_{2}-x_{5}\right)\left(x_{2}-x_{6}\right)\left(x_{5}-x_{6}\right) \times\left(x_{1}-x_{4}\right) \times\left(x_{3}-x_{9}\right) \times x_{7}^{4} x_{8}^{4} .
\end{aligned}
$$

The polynomial $\delta_{T}$ and the monomial $\mathbf{x}(T)$ are related as follows.

Observation 10. The lexicographical leading term of $\delta_{T}$ is $\mathbf{x}(T)$.

\subsection{A generating set for $V_{n, \lambda}$ as a $\mathbb{Q}\left[\mathrm{x}_{n}\right]$-module}

Recall that $\mathbb{Q}\left[\mathbf{x}_{n}\right]$ acts on itself by the rule $f \odot g:=(\partial f)(g)$. The harmonic space $V_{n, \lambda}$ is a submodule for this action. The polynomials $\delta_{T}$, where $T$ varies over $\operatorname{Inj}(\lambda, \leqslant n)$, will turn out to generate the harmonic space $V_{n, \lambda}$ as a $\mathbb{Q}\left[\mathbf{x}_{n}\right]$-module. We first establish that the $\delta_{T}$ are contained in $V_{n, \lambda}$.

Lemma 11. Let $T \in \operatorname{Inj}(\lambda, \leqslant n)$ be a tableau. The polynomial $\delta_{T}$ is contained in the harmonic space $V_{n, \lambda}$.

Proof. It suffices to check that for each generator $f$ of the ideal $I_{n, \lambda}$ we have $f \odot \delta_{T}=0$. If $f=x_{i}^{s}$, the identity $f \odot \delta_{T}=0$ follows from the fact that no exponents $\geqslant s$ appear in $\mathbf{x}(T)$ or in $\delta_{T}$. We may therefore fix $1 \leqslant j \leqslant r$ and assume that $f$ is the elementary symmetric polynomial $f=e_{d}(S)$ for some $S \subseteq[n]$ of size $|S|=n-j+1$ whose degree $d$ satisfies $d>|S|-\lambda_{j}^{\prime}-\lambda_{j+1}^{\prime}-\cdots-\lambda_{n}^{\prime}$. It suffices to show that $e_{d}(S) \odot \delta_{T}=0$. Without loss of generality we assume $d \leqslant|S|$, so that $e_{d}(S) \neq 0$.

We give a combinatorial model for $e_{d}(S) \odot \delta_{T}$ as follows. Let $C_{1}, \ldots, C_{r}$ be the columns of $T$, read from left to right.

The $(S, T)$-staircase consists of $n$ columns of boxes arranged as follows. The $i^{\text {th }}$ column is decorated with the symbol $i_{t}$ where $t=0$ if $i$ does not appear in $T$ and $i \in C_{t}$ otherwise. If $i \notin S$, we further decorate $i_{t}$ with a circle $i_{i}^{\circ}$; such entries $i$ are called frozen. If $i$ does not appear in $T$, the $i^{t h}$ column of the $(S, T)$-staircase has $s-1$ boxes. If $i$ is in row $r$ of $C_{t}$, the $i^{t h}$ column has $\left|C_{t}\right|-r=\lambda_{t}^{\prime}-r$ boxes.

Let us give an example of these concepts. Suppose $n=9, s=5, \lambda=(3,3,1)$, and $T$ is as in (20). Let $j=2$ and take $S=\{1,2,3,4,6,7,8,9\}$ so that $[n]-S=\{5\}$. The $(S, T)$-staircase is as follows. 


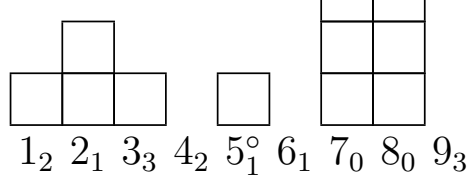

We apply permutations $w \in C_{T}$ to sequences $\left(B_{1}, \ldots, B_{n}\right)$ of $n$ stacks of boxes by rearranging the box stacks. A permuted $(S, T)$-staircase $\sigma$ is obtained from the $(S, T)$ staircase by applying some permutation $w \in C_{T}$ which stabilizes the columns of $T$. If $(S, T)$ is as above and $w=(2,5,6)(3,9) \in C_{T}$, the associated permuted staircase $\sigma$ is

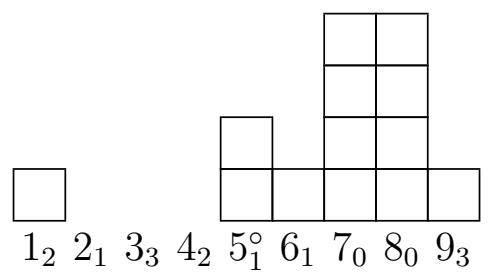

Observe that $w$ leaves the labels unchanged. It should be clear that, for fixed $S$ and $T$, a permuted $(S, T)$-staircase determines the permutation $w \in C_{T}$ uniquely.

The sign of a permuted $(S, T)$-staircase $\sigma$ is the sign of the permutation $w \in C_{T}$, i.e. $\operatorname{sign}(\sigma)=\operatorname{sign}(w)$; in our example $\operatorname{sign}((2,5,6)(3,9))=-1$. The weight $\operatorname{wt}(\sigma)$ of $\sigma$ is the monomial $\operatorname{wt}(\sigma)=x_{1}^{a_{1}} \cdots x_{n}^{a_{n}}$, where $a_{i}$ is the number of boxes in column $i$. In our example $\operatorname{wt}(\sigma)=x_{1} x_{5}^{2} x_{6} x_{7}^{4} x_{8}^{4} x_{9}$. The polynomial $\delta_{T}$ has the combinatorial interpretation

$$
\delta_{T}=\sum_{\sigma} \operatorname{sign}(\sigma) \cdot \operatorname{wt}(\sigma),
$$

where the sum is over all permuted $(S, T)$-staircases $\sigma$.

A d-dotted permuted $(S, T)$-staircase $\sigma^{\bullet}$ is obtained from a permuted $(S, T)$-staircase $\sigma$ by marking $d$ boxes with $\bullet$ so that no two marked boxes are in the same column and so that no frozen column $i_{t}^{\circ}$ gets a marked box. With $d=4$ and $\sigma$ as above, an example choice for $\sigma^{\bullet}$ is as follows.

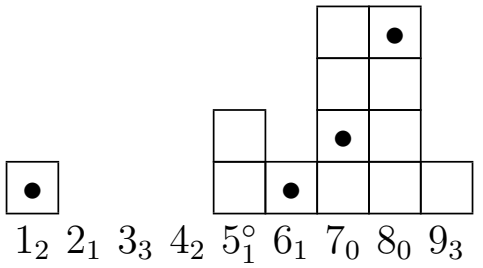

The $\operatorname{sign}$ of $\sigma^{\bullet}$ is the same as the sign of the unmarked $(S, T)$-staircase $\sigma$, i.e. $\operatorname{sign}\left(\sigma^{\bullet}\right)=$ $\operatorname{sign}(\sigma)$. The weight $\operatorname{wt}\left(\sigma^{\bullet}\right)$ is the monomial $x_{1}^{a_{1}} \cdots x_{n}^{a_{n}}$ where $a_{i}$ is the number of unmarked boxes in column $i$; in the above example $\operatorname{wt}\left(\sigma^{\bullet}\right)=x_{5}^{2} x_{7}^{3} x_{8}^{3} x_{9}$.

The polynomial $e_{d}(S) \odot \delta_{T}$ has a combinatorial interpretation in terms of dotted permuted staircases. More precisely we have

$$
e_{d}(S) \odot \delta_{T}=\sum_{\sigma^{\bullet}} \operatorname{sign}\left(\sigma^{\bullet}\right) \cdot \operatorname{wt}\left(\sigma^{\bullet}\right)
$$


where the sum is over all $d$-dotted permuted $(S, T)$-staircases $\sigma^{\bullet}$. Our goal is to show that Equation (25) equals zero.

We use a sign-reversing involution to prove that the right-hand side of Equation (25) vanishes. The following key observation may be verified from our assumptions on $|S|$ and $d$.

Observation: For any d-dotted permuted $(S, T)$-staircase $\sigma^{\bullet}$, there is some value $1 \leqslant t \leqslant r$ such that for the corresponding column $C_{t}$ of $T$ :

1. no column of $\sigma^{\bullet}$ indexed by an entry in $C_{t}$ is frozen, and

2. at least one column of $\sigma^{\bullet}$ indexed by an entry of $C_{t}$ contains a $\bullet$.

In our running example, we may take $t=2$, so that the corresponding column $C_{2}$ of $T$ has entries 1 and 4 . Neither column 1 nor column 4 of $\sigma^{\bullet}$ is frozen and column 1 contains a $\bullet$.

If $\sigma^{\bullet}$ is a $d$-dotted permuted $(S, T)$-staircase, let $t \geqslant 1$ be minimal such that $t$ is as in the above observation. Two of the columns in $\sigma^{\bullet}$ indexed by entries in $C_{t}$ must contain the same number of unmarked boxes. Let $\iota\left(\sigma^{\bullet}\right)$ be obtained from $\sigma^{\bullet}$ by interchanging the two such columns of minimal height. In our running example, we have $t=2$ and $\iota\left(\sigma^{\bullet}\right)$ is given by interchanging columns 1 and 4:

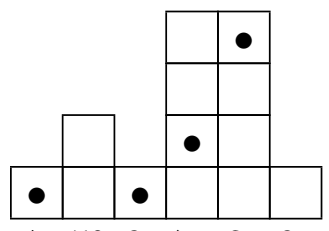

$\begin{array}{llllllllllll}1_{2} & 2_{1} & 3_{3} & 4_{2} & 5_{1}^{\circ} & 6_{1} & 7_{0} & 8_{0} & 9_{3}\end{array}$

For any $d$-dotted permuted $(S, T)$-staircase $\sigma^{\bullet}$ we have

$$
\iota\left(\iota\left(\sigma^{\bullet}\right)\right)=\sigma^{\bullet}, \quad \operatorname{sign}\left(\iota\left(\sigma^{\bullet}\right)\right)=-\operatorname{sign}\left(\sigma^{\bullet}\right), \quad \text { and } \quad \operatorname{wt}\left(\iota\left(\sigma^{\bullet}\right)\right)=\operatorname{wt}\left(\sigma^{\bullet}\right) .
$$

That is, the map $\sigma^{\bullet} \mapsto \iota\left(\sigma^{\bullet}\right)$ is a weight-preserving and sign-reversing involution which verifies that the right-hand side of Equation (25) vanishes.

Definition 12. Let $k \leqslant n$, let $\lambda=\left(\lambda_{1} \geqslant \cdots \geqslant \lambda_{s}\right)$ be a partition of $k$, and let $\sigma \in \mathcal{O P} \mathcal{P}_{n, \lambda}$. Define $T(\sigma) \in \operatorname{Inj}(\lambda, \leqslant n)$ to be the tableau whose $i^{\text {th }}$ column consists of the entries in row $i$ from the top of the container of $\sigma$.

For example, if $\sigma$ is the ordered set partition (11) displayed again here

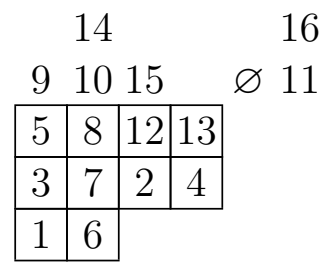

$\begin{array}{llllll}1 & 2 & 3 & 4 & 5 & 6\end{array}$ 
then $T(\sigma)$ is shown below.

$$
\begin{array}{|c|c|c|}
\hline 5 & 2 & 1 \\
\hline 8 & 3 & 6 \\
\hline 12 & 4 & \multicolumn{1}{|}{} \\
\cline { 1 - 2 } 13 & 7 & \multicolumn{1}{|}{} \\
\cline { 1 - 2 } & &
\end{array}
$$

In particular, floating entries in $\sigma$ do not appear in $T(\sigma)$. The most important property of $T(\sigma)$ is shown below; it follows from Observation 10 and the definition of maxcode.

Observation 13. The lexicographical leading term of $\delta_{T(\sigma)}$ is the monomial $x_{1}^{a_{1}} \cdots x_{n}^{a_{n}}$ where $\left(a_{1}, \ldots, a_{n}\right)=\operatorname{maxcode}(\sigma)$.

Observation 13 allows us to associate a harmonic polynomial to any ordered set partition $\sigma \in \mathcal{O P}_{n, \lambda}$.

Definition 14. Let $k \leqslant n$, let $\lambda=\left(\lambda_{1} \geqslant \cdots \geqslant \lambda_{s}\right)$ be a partition of $k$, and let $\sigma \in \mathcal{O P}_{n, \lambda}$ satisfy $\operatorname{code}(\sigma)=\left(c_{1}, \ldots, c_{n}\right)$ and $\max \operatorname{code}(\sigma)=\left(a_{1}, \ldots, a_{n}\right)$. Define a polynomial $\delta_{\sigma} \in$ $\mathbb{Q}\left[\mathbf{x}_{n}\right]$ by

$$
\delta_{\sigma}:=\left(x_{1}^{a_{1}-c_{1}} \cdots x_{n}^{a_{n}-c_{n}}\right) \odot \delta_{T(\sigma)} .
$$

We are ready to describe our generating set for the harmonic space $V_{n, \lambda}$ as a $\mathbb{Q}\left[\mathbf{x}_{n}\right]$ module.

Theorem 15. Let $k \leqslant n$ be positive integers and let $\lambda=\left(\lambda_{1} \geqslant \cdots \geqslant \lambda_{s}\right)$ be a partition of $k$. The harmonic space $V_{n, \lambda}$ is the smallest subspace of $\mathbb{Q}\left[\mathbf{x}_{n}\right]$ which

- contains the polynomial $\delta_{T}$ for every tableau $T \in \operatorname{Inj}(\lambda, \leqslant n)$, and

- is closed under the partial derivative operators $\partial / \partial x_{1}, \ldots, \partial / \partial x_{n}$.

Equivalently, the set $\left\{\delta_{T}: T \in \operatorname{Inj}(\lambda, \leqslant n)\right\}$ generates $V_{n, \lambda}$ as a $\mathbb{Q}\left[\mathbf{x}_{n}\right]$-module.

Proof. Let $W_{n, \lambda}$ be the subspace defined by the two bullet points of the theorem. By Lemma 11 we have the containment of vector spaces

$$
W_{n, \lambda} \subseteq V_{n, \lambda}
$$

We also have

$$
\operatorname{dim} V_{n, \lambda}=\left|\mathcal{C}_{n, \lambda}\right|=\left|\mathcal{O} \mathcal{P}_{n, \lambda}\right|,
$$

where the first equality follows from Theorem 4 and the second follows from Theorem 8 . It therefore suffices to exhibit $\left|\mathcal{O P} \mathcal{P}_{n, \lambda}\right|$ linearly independent elements of $W_{n, \lambda}$.

Indeed, Observation 13 and Definition 14 imply that the lexicographical leading term of $\delta_{\sigma}$ has exponent sequence given by code $(\sigma)$. Theorem 8 guarantees that the set $\left\{\delta_{\sigma}\right.$ : $\left.\sigma \in \mathcal{O P}_{n, \lambda}\right\}$ is linearly independent and Lemma 11 assures that the polynomials in this set lie in $W_{n, \lambda}$.

The proof of Theorem 15 also yields a harmonic basis of $R_{n, \lambda}$. 
Theorem 16. Let $k \leqslant n$ be positive integers and let $\lambda=\left(\lambda_{1} \geqslant \cdots \geqslant \lambda_{s}\right)$ be a partition of $k$. The set

$$
\left\{\delta_{\sigma}: \sigma \in \mathcal{O P}_{n, \lambda}\right\}
$$

is a harmonic basis of $R_{n, \lambda}$.

Recall that the Hilbert series of a graded $\mathbb{Q}$-algebra $R=\bigoplus_{d \geqslant 0} R_{d}$ with each graded piece finite-dimensional is the formal power series

$$
\operatorname{Hilb}(R ; q):=\sum_{d \geqslant 0}\left(\operatorname{dim} R_{d}\right) \cdot q^{d}
$$

We have a combinatorial expression for the Hilbert series of $R_{n, \lambda}$.

Corollary 17. Let $k \leqslant n$ be positive integers and let $\lambda=\left(\lambda_{1} \geqslant \cdots \geqslant \lambda_{s}\right)$ be a partition of $k$. We have

$$
\operatorname{Hilb}\left(R_{n, \lambda} ; q\right)=\sum_{\sigma \in \mathcal{O P}_{n, \lambda}} q^{\operatorname{coinv}(\sigma)}
$$

Proof. The degree of the polynomial $\delta_{\sigma}$ is $\operatorname{coinv}(\sigma)$. Now apply Theorem 16. Alternatively, combine Theorems 4 and 8.

Remark 18. Griffin found [5, Thm. 5.12] a variant of the coinv statistic which encodes the graded $\mathfrak{S}_{n}$-isomorphism type of $R_{n, \lambda}$. This may be viewed as a moral extension of Corollary 17. Recall that the graded Frobenius image $\operatorname{grFrob}(V ; q)$ of a graded $\mathfrak{S}_{n}$-module $V=\bigoplus_{d \geqslant 0} V_{d}$ is the symmetric function

$$
\operatorname{grFrob}(V ; q)=\sum_{d \geqslant 0} q^{d} \sum_{\rho \vdash n}\left(\text { multiplicity of the } \mathfrak{S}_{n} \text {-irreducible } S^{\rho} \text { in } V_{d}\right) \cdot s_{\rho}(\mathbf{x})
$$

where $s_{\rho}(\mathbf{x})$ is the Schur function corresponding to the partition $\rho \vdash n$.

Given $n$ and $\lambda=\left(\lambda_{1} \geqslant \cdots \geqslant \lambda_{s}\right)$ with $\lambda_{s} \geqslant 0$, Griffin considers the family ECI E $_{n, \lambda}$ of extended column-increasing fillings. These are sequences $\mu=\left(M_{1}|\cdots| M_{s}\right)$ of $s$ multisets of positive integers in which $\left|M_{i}\right| \geqslant \lambda_{i}$ for all $i$ such that $\left|M_{1}\right|+\cdots+\left|M_{s}\right|=n$. Elements of $\mathrm{ECI}_{n, \lambda}$ may be viewed as fillings of the container diagram of $\lambda$ which weakly increase going up columns and fill every box in the container; as an example, the element

$$
\mu=(1,1,2,4|2,4,4,5,6| 1,2,4|3,3| \varnothing \mid 1,1) \in \mathrm{ECI}_{16,(3,3,2,2,0,0), 6}
$$

is shown below.

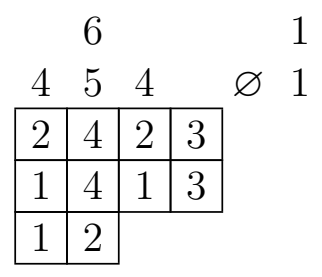


Given $\mu \in \mathrm{ECI}_{n, \lambda}$, we attach the monomial $\mathbf{x}^{\mu}$ in the variable set $\mathbf{x}=\left(x_{1}, x_{2}, \ldots\right)$ where the exponent of $x_{i}$ is the multiplicity of $i$ in $\mu$. For $\mu$ as above, we have $\mathbf{x}^{\mu}=x_{1}^{5} x_{2}^{3} x_{3}^{2} x_{4}^{4} x_{5} x_{6}$. Griffin defines a statistic inv on $\mathrm{ECI}_{n, \lambda}$ which is similar to (but not quite the same as) our statistic coinv - see [5, Rmk. 5.3] - and proves that the graded Frobenius image of $R_{n, \lambda}$ is given combinatorially by

$$
\operatorname{grFrob}\left(R_{n, \lambda} ; q\right)=\sum_{\mu \in \mathrm{ECI}_{n, \lambda}} q^{\operatorname{inv}(\mu)} \mathbf{x}^{\mu}
$$

\subsection{A conjecture of Wilson}

For positive integers $n, k$, and $s$, the following ideal $I_{n, k, s} \subseteq \mathbb{Q}\left[\mathbf{x}_{n}\right]$ was introduced in [8]:

$$
I_{n, k, s}:=\left\langle e_{n}\left(\mathbf{x}_{n}\right), e_{n-1}\left(\mathbf{x}_{n}\right), \ldots, e_{n-k+1}\left(\mathbf{x}_{n}\right), x_{1}^{s}, x_{2}^{s}, \ldots, x_{n}^{s}\right\rangle .
$$

Let $R_{n, k, s}:=\mathbb{Q}\left[\mathbf{x}_{n}\right] / I_{n, k, s}$ be the corresponding quotient ring.

When $k \leqslant s$, we have $R_{n, k, s}=R_{n, \lambda}$ where $\lambda=\left(1^{k}, 0^{s-k}\right)$ and the structure of $R_{n, k, s}$ as a graded $\mathfrak{S}_{n}$-module was determined by Haglund-Rhoades-Shimozono [8]. Indeed, the ring $R_{n, k, s}$ was one of the motivating examples for defining $R_{n, \lambda}$ for general $\lambda$.

When $k>s$, the structure of $R_{n, k, s}$ was not studied in [8] because the Gröbner theory of the ideal $I_{n, k, s}$ was more complicated in this case. Although it is not immediately obvious, we will establish that $R_{n, k, s}$ is also an instance of the $R_{n, \lambda}$ rings when $k>s$.

For positive integers $k$ and $s$, write $k=q s+r$ for integers $q, r \geqslant 0$ with $r<s$. We define $\lambda(k, s):=\left((q+1)^{r}, q^{s-r}\right)$ to be the partition of $k$ given by $r$ copies of $q+1$ followed by $s-r$ copies of $q$.

Proposition 19. For any positive integers $n, k, s$ we have $I_{n, k, s}=I_{n, \lambda}$ and $R_{n, k, s}=R_{n, \lambda}$ where $\lambda=\lambda(k, s)$.

Proposition 19 implies that $\operatorname{dim} R_{n, k, s}=\left|\mathcal{O P}_{n, \lambda(k, s)}\right|$. This combinatorial expression for $\operatorname{dim} R_{n, k, s}$ was conjectured by Andy Wilson (personal communication).

Proof. Let $\lambda=\lambda(k, s)$. Every generator of $I_{n, k, s}$ is also a generator of $I_{n, \lambda}$, so we have the containment of ideals

$$
I_{n, k, s} \subseteq I_{n, \lambda}
$$

It therefore suffices to show that every generator of $I_{n, \lambda}$ lies in $I_{n, k, s}$. The generators $x_{i}^{s}$ of $I_{n, \lambda}$ are also generators of $I_{n, k, s}$, so it suffices to check that the elementary symmetric polynomials $e_{d}(S)$ in partial variable sets $S \subseteq[n]$ which appear as generators of $I_{n, \lambda}$ lie in $I_{n, k, s}$.

Let $S \subseteq[n]$ and $1 \leqslant d \leqslant n$ be such that $e_{d}(S)$ is a generator of $I_{n, \lambda}$. We prove that $e_{d}(S) \in I_{n, k, s}$ by descending induction on the size $|S|$ of $S$. If $|S|=n$, we have $e_{d}(S)=e_{d}\left(\mathbf{x}_{n}\right)$ and $d>n-k$, so that $e_{d}(S)$ is a generator of $I_{n, k, s}$. 
If $|S|<n$, choose an arbitrary index $i \in[n]-S$ and let $T=S \cup\{i\}$. The polynomial $e_{d}(S)$ may be expressed as

$$
\begin{aligned}
& e_{d}(S)=e_{d}(T)-x_{i} e_{d-1}(S)=e_{d}(T)-x_{i} e_{d-1}(T)+x_{i}^{2} e_{d-2}(S) \\
&=\cdots=\left[\sum_{j=0}^{s-1}(-1)^{j} x_{i}^{j} e_{d-j}(T)\right]+(-1)^{s} x_{i}^{s} e_{d-s}(S) .
\end{aligned}
$$

We claim that each term in the sum on the right-hand side of Equation (37) lies in $I_{n, k, s}$, so that $e_{d}(S) \in I_{n, k, s}$. Indeed, since $e_{d}(S)$ is a generator of $I_{n, \lambda}$, each column aside from the shortest column of $\lambda$ has $s$ boxes, and $T$ has one more element than $S$, each of the $s$ polynomials $e_{d}(T), e_{d-1}(T), \ldots, e_{d-s+1}(T)$ are generators of $I_{n, \lambda}$, and so lie in $I_{n, k, s}$ by induction. In particular, the sum in the square brackets lies in $I_{n, k, s}$. Furthermore, the monomial $x_{i}^{s}$ is a generator of $I_{n, k, s}$, so that $(-1)^{s} x_{i}^{s} e_{d-s}(S) \in I_{n, k, s}$. We conclude that $e_{d}(S) \in I_{n, k, s}$, finishing the proof.

\subsection{The lexicographical leading terms of harmonic polynomials}

Let $<$ be the lexicographical order on monomials in $\mathbb{Q}\left[\mathbf{x}_{n}\right]$. That is, we have $x_{1}^{a_{1}} \cdots x_{n}^{a_{n}}<$ $x_{1}^{b_{1}} \cdots x_{n}^{b_{n}}$ if and only if there is some $1 \leqslant i \leqslant n$ such that $a_{1}=b_{1}, \ldots, a_{i-1}=b_{i-1}$, and $a_{i}<b_{i}$. It is well known that the lexicographical order $<$ is a monomial order, meaning that

- we have $1 \leqslant m$ for any monomial $m$ in $x_{1}, \ldots, x_{n}$ and

- given three monomials $m, m^{\prime}, m^{\prime \prime}$ with $m \leqslant m^{\prime}$, we have $m \cdot m^{\prime \prime} \leqslant m^{\prime} \cdot m^{\prime \prime}$.

If $f \in \mathbb{Q}\left[\mathbf{x}_{n}\right]$ is a nonzero polynomial, let $\operatorname{in}_{<}(f)$ denote the largest monomial in lexicographical order appearing in $f$.

The coinversion codes $\mathcal{C}_{n, \lambda}$ of ordered set partitions in $\mathcal{O} \mathcal{P}_{n, \lambda}$ are precisely the exponent sequences of the lexicographical leading monomials of nonzero polynomials in $V_{n, \lambda}$. This gives another connection between harmonic polynomials and ordered set partitions.

Theorem 20. Let $k \leqslant n$ be positive integers and let $\lambda=\left(\lambda_{1} \geqslant \cdots \geqslant \lambda_{s}\right)$ be a partition of $k$ into $s$ nonnegative parts. If $f \in V_{n, \lambda}$ is any nonzero harmonic polynomial with $\operatorname{in}_{<} f=x_{1}^{c_{1}} x_{2}^{c_{2}} \cdots x_{n}^{c_{n}}$ then $\left(c_{1}, c_{2}, \ldots, c_{n}\right) \in \mathcal{C}_{n, \lambda}$.

Proof. The proof is by induction on $n$. If $n=1$, then $\lambda=\left(0^{s}\right)$ or $\lambda=\left(1,0^{s-1}\right)$. If $\lambda=\left(0^{s}\right)$ then $I_{1,\left(0^{s}\right)}=\left\langle x_{1}^{s}\right\rangle \subseteq \mathbb{Q}\left[x_{1}\right]$ so that $V_{1,\left(0^{s}\right)}=\operatorname{span}_{\mathbb{Q}}\left\{1, x_{1}, x_{1}^{2}, \ldots, x_{1}^{s-1}\right\}$. Since $\mathcal{C}_{1,\left(0^{s}\right)}=\{(0),(1),(2), \ldots,(s-1)\}$, the result is true in this case. If $\lambda=\left(1,0^{s-1}\right)$ then $I_{1,\left(1,0^{s-1}\right)}=\left\langle x_{1}\right\rangle \subseteq \mathbb{Q}\left[x_{1}\right]$ so that $V_{1,\left(1,0^{s-1}\right)}=\mathbb{Q}$. Since $\mathcal{C}_{1,\left(1,0^{s-1}\right)}=\{(0)\}$, this completes the proof when $n=1$.

When $n$ is arbitrary and $\lambda=\left(0^{s}\right)$ is a partition of $k=0$, we may compute directly that

$$
V_{n,\left(0^{s}\right)}=\operatorname{span}_{\mathbb{Q}}\left\{x_{1}^{a_{1}} \cdots x_{n}^{a_{n}}: a_{1}, \ldots, a_{n}<s\right\}
$$


from which the theorem follows in this case. We therefore assume going forward that $n, k>0$.

Our main tool will be Griffin's disjoint union decomposition (18) of $\mathcal{C}_{n, \lambda}$ according to the first terms of its sequences, recapitulated here for convenience. Recall that $\ell(\lambda)$ is the number of nonzero parts of $\lambda$.

$$
\begin{aligned}
\mathcal{C}_{n, \lambda}=\bigsqcup_{i=1}^{\ell(\lambda)}\left\{\left(i-1, c_{2}, \ldots, c_{n}\right):\left(c_{2}, \ldots, c_{n}\right) \in \mathcal{C}_{n-1, \lambda\left(\lambda^{(i)}\right.}\right\} \sqcup \\
\qquad \bigsqcup_{j=\ell(\lambda)+1}^{s}\left\{\left(j-1, c_{2}, \ldots, c_{n}\right):\left(c_{2}, \ldots, c_{n}\right) \in \mathcal{C}_{n-1, \lambda}\right\}
\end{aligned}
$$

Let $f \in V_{n, \lambda}$ be a nonzero polynomial. Since $f$ is harmonic and $x_{1}^{s} \in V_{n, \lambda}$, we have

$$
x_{1}^{s} \odot f=\partial^{s} f / \partial x_{1}^{s}=0 .
$$

In particular, if $\operatorname{in}_{<}(f)=x_{1}^{c_{1}} x_{2}^{c_{2}} \cdots x_{n}^{c_{n}}$ then $c_{1}<s$ and, by the definition of $<$, no monomial appearing in $f$ has an exponent of $x_{1}$ greater than $c_{1}$. In particular, the polynomial $x_{1}^{c_{1}} \odot f$ does not involve the variable $x_{1}$. Since the lexicographical order is a monomial order, we have

$$
\operatorname{in}_{<}(f)=x_{1}^{c_{1}} \cdot \operatorname{in}_{<}\left(x_{1}^{c_{1}} \odot f\right) .
$$

Let $W_{n-1, \lambda^{\left(c_{1}+1\right)}} \subseteq \mathbb{Q}\left[x_{2}, x_{3}, \ldots, x_{n}\right]$ be the image under the algebra map $x_{i} \mapsto x_{i+1}$ of the subspace $V_{n-1, \lambda}\left(c_{1}+1\right) \subseteq \mathbb{Q}\left[x_{1}, x_{2}, \ldots, x_{n-1}\right]$. Similarly, let $W_{n-1, \lambda}$ be the image under $x_{i} \mapsto x_{i+1}$ of $V_{n-1, \lambda}$. Thanks to (38), (40), and induction, it suffices to prove the following claim.

Claim: If $c_{1}<\ell(\lambda)$ then $x_{1}^{c_{1}} \odot f \in W_{n-1, \lambda}\left(c_{1}+1\right)$. If $\ell(\lambda) \leqslant c_{1}<s$ then $x_{1}^{c_{1}} \odot f \in W_{n-1, \lambda}$.

Let $J_{n-1, \lambda\left(c_{1}\right)}, J_{n-1, \lambda} \subseteq \mathbb{Q}\left[x_{2}, x_{3}, \ldots, x_{n}\right]$ be the images under the algebra map $x_{i} \mapsto$ $x_{i+1}$ of $I_{n-1, \lambda\left(c_{1}\right)}, I_{n, \lambda} \subseteq \mathbb{Q}\left[x_{1}, x_{2}, \ldots, x_{n-1}\right]$. We verify that $x_{1}^{c_{1}} \odot f$ is annihilated by the generators of the relevant $J$-ideal. For the generators of the form $x_{i}^{s}$ this is clear, so we need only verify this statement for generators of the form $e_{d}(S)$.

The proof of our Claim hinges on the following observation.

Observation: Let $S \subseteq\{2,3, \ldots, n\}$ and write $S^{\prime}:=S \cup\{1\}$. Let $d \geqslant 1$ be such that $e_{d}(S)$ appears as a generator of $J_{n-1, \lambda\left(c_{1}+1\right)}$ (in the case $c_{1}<\ell(\lambda)$ ) or $J_{n-1, \lambda}$ (in the case $\left.\ell(\lambda) \leqslant c_{1}<s\right)$. Then $e_{d+1}\left(S^{\prime}\right)$ appears as a generator of $I_{n, \lambda}$ so that $e_{d+1}\left(S^{\prime}\right) \odot f=0$.

Let $d \geqslant 1$ and $S \subseteq\{2,3, \ldots, n\}$ be such that $e_{d}(S)$ is a nonzero generator of the $J$-ideal described in the Observation. Our analysis breaks up into cases depending on the value of $c_{1}$.

Case 1: We have $c_{1}>0$.

In this case we compute

$$
\begin{aligned}
e_{d}(S) \odot\left(x_{1}^{c_{1}} \odot f\right)=\left(x_{1}^{c_{1}} e_{d}(S)\right) \odot f=\left(x_{1}^{c_{1}-1} e_{d+1}\left(S^{\prime}\right)-x_{1}^{c_{1}-1} e_{d+1}(S)\right) \odot f \\
=x_{1}^{c_{1}-1} e_{d+1}\left(S^{\prime}\right) \odot f-x_{1}^{c_{1}-1} e_{d+1}(S) \odot f .
\end{aligned}
$$


By our Observation, we have $e_{d+1}\left(S^{\prime}\right) \odot f=0$, so the first term on the right-hand side of Equation (41) vanishes and we have

$$
e_{d}(S) \odot\left(x_{1}^{c_{1}} \odot f\right)=-x_{1}^{c_{1}-1} e_{d+1}(S) \odot f .
$$

We may now show $e_{d}(S) \odot\left(x_{1}^{c_{1}} \odot f\right)=0$ by descending induction on $d$. In the base case $d=|S|$, we have $e_{d+1}(S)=0$ so this follows from Equation (42). When $d<|S|$, the polynomial $e_{d+1}(S)$ is a nonzero generator of the $J$-ideal described in the Observation, so that $e_{d+1}(S) \odot f=0$ by induction, so Equation (42) shows $e_{d}(S) \odot\left(x_{1}^{c_{1}} \odot f\right)=0$.

Case 2: We have $c_{1}=0$.

Here is we make use of our assumption that $\lambda$ is a partition of a positive integer $k$ so that $\lambda_{c_{1}+1}=\lambda_{1}>0$. We compute

$$
e_{d}(S) \odot\left(x_{1}^{c_{1}} \odot f\right)=e_{d}(S) \odot f=e_{d}\left(S^{\prime}\right) \odot f .
$$

The second equality is true because $f$ does not involve the variable $x_{1}$ so that every monomial appearing in $e_{d}\left(S^{\prime}\right)$ involving $x_{1}$ annihilates $f$. Since $\lambda^{\left(c_{1}+1\right)}=\lambda^{(1)}$ is the non-decreasing rearrangement of $\left(\lambda_{1}-1, \lambda_{2}, \ldots, \lambda_{s}\right)$ and $e_{d}(S)$ is a nonzero generator of $J_{n-1, \lambda^{(1)}}$, we see that $e_{d}\left(S^{\prime}\right)$ is a nonzero generator of $I_{n, \lambda}$ so that the right-hand side of Equation (43) vanishes.

Theorem 20 directly gives the upper bound

$$
\operatorname{dim} V_{n, \lambda} \leqslant\left|\mathcal{C}_{n, \lambda}\right|
$$

on the dimension of the harmonic space $V_{n, \lambda}$. Indeed, if $N>\left|\mathcal{C}_{n, \lambda}\right|$ and we are given $N$ harmonic polynomials $f_{1}, f_{2}, \ldots, f_{N} \in V_{n, \lambda}$, there exist $c_{1}, c_{2}, \ldots, c_{N} \in \mathbb{Q}$ not all zero so that

for any monomial $m$ whose exponent sequence appears in $\mathcal{C}_{n, \lambda}$, the coefficient of $m$ in $f:=c_{1} f_{1}+c_{2} f_{2}+\cdots+c_{N} f_{N}$ is zero.

But $f \in V_{n, \lambda}$ is harmonic, so Theorem 20 forces $f=0$, implying that $f_{1}, f_{2}, \ldots, f_{N}$ are linearly dependent. Of course, Equation (44) also follows from Griffin's [5] result $\operatorname{dim} R_{n, \lambda}=\left|\mathcal{C}_{n, \lambda}\right|$ and the vector space isomorphism between the quotient ring and harmonic space of a given ideal.

\section{Conclusion}

Let $k \leqslant n$ be positive integers and let $\lambda \vdash k$. In this paper we studied the harmonic space $V_{n, \lambda}$ associated to the ring $R_{n, \lambda}$ using polynomials $\delta_{T}$ and $\delta_{\sigma}$ closely related to the Vandermonde determinant $\delta_{n} \in \mathbb{Q}\left[\mathbf{x}_{n}\right]$. In the case $\lambda_{1} \leqslant 1$, Rhoades and Wilson [13] gave an alternate harmonic-like model for $R_{n, \lambda}$ involving anticommuting variables as follows.

Superspace of rank $n$ is the $\mathbb{Q}$-algebra $\Omega_{n}$ given by a tensor product

$$
\Omega_{n}:=\mathbb{Q}\left[x_{1}, \ldots, x_{n}\right] \otimes \wedge\left\{\theta_{1}, \ldots, \theta_{n}\right\}
$$


of a rank $n$ polynomial ring (with generators $x_{1}, \ldots, x_{n}$ ) with a rank $n$ exterior algebra (with generators $\theta_{1}, \ldots, \theta_{n}$ ). The terminology here comes from physics where the $x_{i}$ model the positions of bosons and the $\theta_{i}$ model the positions of fermions.

The symmetric group $\mathfrak{S}_{n}$ acts on $\Omega_{n}$ by permuting the $x_{i}$ and $\theta_{i}$ simultaneously. The differentiation action $f \odot g$ of $\mathbb{Q}\left[\mathbf{x}_{n}\right]$ on itself extends to an action $\mathbb{Q}\left[\mathbf{x}_{n}\right] \otimes \Omega_{n} \rightarrow \Omega_{n}$ of $\mathbb{Q}\left[\mathbf{x}_{n}\right]$ on superspace by acting on the first tensor factor.

Let $\varepsilon_{n}:=\sum_{w \in \mathfrak{S}_{n}} \operatorname{sign}(w) \cdot w$ be the antisymmetrizing element of $\mathbb{Q}\left[\mathfrak{S}_{n}\right]$. For any $r \leqslant n$ and any length $r$ sequence $\mathbf{a}=\left(a_{1}, \ldots, a_{r}\right)$ of nonnegative integers, the a-superspace Vandermonde was defined in [13] to be the following element of $\Omega_{n}$ :

$$
\delta_{n}(\mathbf{a}):=\varepsilon_{n} \cdot\left(x_{1}^{a_{1}} x_{2}^{a_{2}} \cdots x_{r}^{a_{r}} x_{r+1}^{n-r-1} \cdots x_{n-1}^{1} x_{n}^{0} \times \theta_{1} \theta_{2} \cdots \theta_{r}\right) .
$$

This reduces to the usual Vandermonde when $\mathbf{a}=\varnothing$ is the empty sequence. Rhoades and Wilson [13] defined $V_{n}(\mathbf{a})$ to be the smallest subspace of $\Omega_{n}$ containing $\delta_{n}(\mathbf{a})$ which is closed under the differentiation action of $\mathbb{Q}\left[\mathbf{x}_{n}\right]$. The vector space $V_{n}(\mathbf{a})$ is a graded $\mathfrak{S}_{n}$-module.

Theorem 21. (Rhoades-Wilson [13]) Let $k \leqslant n$ be positive integers and let $\lambda=\left(1^{k}, 0^{s-k}\right)$ be the partition with $k$ copies of 1 and $s-k$ copies of 0 , for some $s \geqslant k$. Let $\mathbf{a}=\left((s-1)^{n-k}\right)$ be the constant sequence with $n-k$ copies of $s-1$.

The graded $\mathfrak{S}_{n}$-module $V_{n}(\mathbf{a})$ is isomorphic to $R_{n, \lambda}$ after grading reversal and twisting by the sign representation.

Question 22. By considering more general parameters a, can the superspace Vandermondes $\delta_{n}(\mathbf{a})$ be used to give models for the quotient rings $R_{n, \lambda}$ for more general partitions $\lambda$ as subspaces of $\Omega_{n}$ ?

In the situation of Theorem 21 when $k=s$, Rhoades and Wilson defined [13] an extension $\mathbb{V}_{n}(\mathbf{a})$ of the module $V_{n}(\mathbf{a})$ by introducing $n$ new commuting variables $y_{1}, \ldots, y_{n}$ and closing under polarization operators. The space $\mathbb{V}_{n}(\mathbf{a})$ is a doubly graded $\mathfrak{S}_{n}$-module, and it was conjectured [13] that its bigraded character is given by the symmetric function $\Delta_{e_{k-1}}^{\prime} e_{n}$ appearing in the Delta Conjecture [7] of Haglund, Remmel, and Wilson. A solution to Question 22 could lead to representation-theoretic models for more general delta operators $\Delta_{s_{\lambda}}^{\prime}$ corresponding to Schur functions $s_{\lambda}$ (see [9] for more details on these operators).

\section{Acknowledgements}

The authors are grateful to Sean Griffin and Andy Wilson for many helpful conversations, as well as to an anonymous referee for their helpful comments. This project was performed as a Research Experience for Undergraduates at UC San Diego in 2019-2020.

\section{References}

[1] E. Artin. Galois Theory, Second edition. Notre Dame Math Lectures, no. 2. Notre Dame: University of Notre Dame, 1944. 
[2] N. Bergeron and A. Garsia. On certain spaces of harmonic polynomials. In: $H y$ pergeometric functions on domains of positivity, Jack polynomials, and applications (Tampa, FL, 1991), 51-86, Contemp. Math. 138, Amer. Math. Soc., Providence, RI, 1992.

[3] W. Fulton. Young Tableaux. Cambridge University Press. Cambridge, 1997.

[4] A. M. Garsia and C. Procesi. On certain graded $S_{n}$-modules and the $q$-Kostka polynomials. Adv. Math., 94 (1) (1992), 82-138.

[5] S. Griffin. Ordered set partitions, Garsia-Procesi modules, and rank varieties. Ph.D. Dissertation, University of Washington, 2020.

[6] J. Haglund, M. Haiman, and N. Loehr. A combinatorial formula for the Macdonald polynomials. J. Amer. Math. Soc., 18 (2005), 735-761.

[7] J. Haglund, J. Remmel, and A. T. Wilson. The Delta Conjecture. Trans. Amer. Math. Soc., 370 (2018), 4029-4057.

[8] J. Haglund, B. Rhoades, and M. Shimozono. Ordered set partitions, generalized coinvariant algebras, and the Delta Conjecture. Adv. Math., 329 (2018), 851-915.

[9] J. Haglund, B. Rhoades, and M. Shimozono. Hall-Littlewood expansions of Schur delta operators at $t=0$. Sém. Loth. Comb., B79c, (2019). (20 pp.)

[10] B. Pawlowski and B. Rhoades. A flag variety for the Delta Conjecture. Trans. Amer. Math. Soc., 372 (2019), 8195-8248.

[11] B. Rhoades. Ordered set partition statistics and the Delta Conjecture. J. Combin. Theory Ser. A, 154 (2018), 172-217.

[12] B. Rhoades and A. T. Wilson. Line configurations and $r$-Stirling partitions. J. Comb., 10 (3) (2019), 411-431.

[13] B. Rhoades and A. T. Wilson. Vandermondes in superspace. Trans. Amer. Math. Soc., 373 (2020), 4483-4516.

[14] T. Tanisaki. Defining ideals of the closures of conjugacy classes and representations of the Weyl groups. Tohoku Math. J. 33 (4) (1982), 575-585. 